\title{
Networks formed from interdependent networks
}

\author{
Jianxi Gao ${ }^{1,2}$, Sergey V. Buldyrev ${ }^{3}$, H. Eugene Stanley and Shlomo Havlin ${ }^{4 \star}$
}

\begin{abstract}
Complex networks appear in almost every aspect of science and technology. Although most results in the field have been obtained by analysing isolated networks, many real-world networks do in fact interact with and depend on other networks. The set of extensive results for the limiting case of non-interacting networks holds only to the extent that ignoring the presence of other networks can be justified. Recently, an analytical framework for studying the percolation properties of interacting networks has been developed. Here we review this framework and the results obtained so far for connectivity properties of 'networks of networks' formed by interdependent random networks.
\end{abstract}

\section{T} he interdisciplinary field of network science has attracted a great deal of attention in recent years ${ }^{1-30}$. This development is based on the enormous number of data that are now routinely being collected, modelled and analysed, concerning social ${ }^{31-39}$, economic $^{14,36,40,41}$, technological ${ }^{40,42-48}$ and biological ${ }^{9,13,49,50}$ systems. The investigation and growing understanding of this extraordinary volume of data will enable us to make the infrastructures we use in everyday life more efficient and more robust.

The original model of networks, random graph theory, was developed in the 1960s by Erdős and Rényi, and is based on the assumption that every pair of nodes is randomly connected with the same probability, leading to a Poisson degree distribution. In parallel, in physics, lattice networks, where each node has exactly the same number of links, have been studied to model physical systems. Although graph theory is a well-established tool in the mathematics and computer science literature, it cannot describe well modern, real-life networks. Indeed, the pioneering 1999 observation by Barabasi $^{2}$, that many real networks do not follow the Erdös-Rényi model but that organizational principles naturally arise in most systems, led to an overwhelming accumulation of supporting data, new models and computational and analytical results, and to the emergence of a new science, that of complex networks.

Complex networks are usually non-homogeneous structures that in many cases obey a power-law form in their degree (that is, number of links per node) distribution. These systems are called scale-free networks. Real networks that can be approximated as scale-free networks include the Internet ${ }^{3}$, the World Wide $\mathrm{Web}^{4}$, social networks ${ }^{31-39}$ representing the relations between individuals, infrastructure networks such as those of airlines ${ }^{51}$, networks in biology $y^{9,13,49,50}$, in particular networks of proteinprotein interactions ${ }^{10}$, gene regulation and biochemical pathways, and networks in physics, such as polymer networks or the potentialenergy-landscape network. The discovery of scale-free networks led to a re-evaluation of the basic properties of networks, such as their robustness, which exhibit a drastically different character than those of Erdős-Rényi networks. For example, whereas homogeneous Erdős-Rényi networks are extremely vulnerable to random failures, heterogeneous scale-free networks are remarkably robust ${ }^{4,5}$. A great part of our current knowledge on networks is based on ideas borrowed from statistical physics, such as percolation theory, fractals and scaling analysis. An important property of these infrastructures is their stability, and it is thus important that we understand and quantify their robustness in terms of node and link failures. Percolation theory was introduced to study network stability and predicted the critical percolation threshold ${ }^{5}$. The robustness of a network is usually either characterized by the value of the critical threshold analysed using percolation theory ${ }^{52}$ or defined as the integrated size of the largest connected cluster during the entire attack process ${ }^{53}$. The percolation approach was also proved to be extremely useful in addressing other scenarios, such as efficient attacks or immunization ${ }^{6,7,54,55}$, and for obtaining optimal paths ${ }^{56}$ as well as for designing robust networks ${ }^{53}$. Network concepts have also proven to be useful for the analysis and understanding of the spread of epidemics ${ }^{57,58}$, and the organizational laws of social interactions, such as friendships $s^{59,60}$ or scientific collaborations $s^{61,62}$. Ref. 63 investigated topologically biased failure in scale-free networks network and control of the robustness or fragility through fine-tuning of the topological bias in the failure process.

A large number of new measures and methods have been developed to characterize network properties, including measures of node clustering, network modularity, correlation between degrees of neighbouring nodes, measures of node importance and methods for the identification and extraction of community structures. These measures demonstrated that many real networks, and in particular biological networks, contain network motifssmall specific subnetworks-that occur repeatedly and provide information about functionality ${ }^{9}$. Dynamical processes, such as flow and electrical transport in heterogeneous networks, were shown to be significantly more efficient when compared with Erdős-Rényi networks ${ }^{64,65}$. Furthermore, it was shown that networks can also possess self-similar properties, so that under proper coarse graining (or, renormalization) of the nodes the network properties remain invariant ${ }^{19}$.

However, these complex systems were mainly modelled and analysed as single networks that do not interact with or depend on other networks. In interacting networks, the failure of nodes in one network generally leads to the failure of dependent nodes in other networks, which in turn may cause further damage to the first network, leading to cascading failures and catastrophic consequences. It is known, for example, that blackouts in various countries have been the result of cascading failures between interdependent systems such as communication and power grid systems $s^{67,68}$. Furthermore, different kinds of critical infrastructure are also coupled together, such as systems of water and food supply, communications, fuel, financial transactions and power generation and transmission. Modern technology has

\footnotetext{
${ }^{1}$ Center for Polymer Studies and Department of Physics, Boston University, Boston, Massachusetts 02215, USA, ${ }^{2}$ Department of Automation, Shanghai Jiao Tong University, 800 Dongchuan Road, Shanghai 200240, China, ${ }^{3}$ Department of Physics, Yeshiva University, New York, New York 10033, USA, ${ }^{4}$ Department of Physics, Bar-Ilan University, 52900 Ramat-Gan, Israel. *e-mail: havlin@ophir.ph.biu.ac.il.
} 


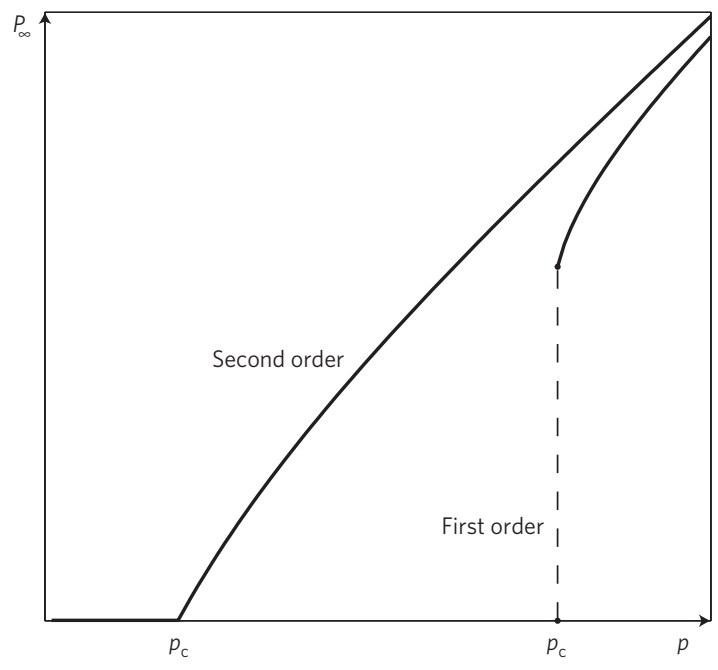

Figure 1 | Schematic demonstration of first- and second-order percolation transitions. In the second-order case, the giant component is continuously approaching zero at the percolation threshold $p=p_{\mathrm{c}}$. In the first-order case, the giant component approaches zero discontinuously.

produced infrastructures that are becoming more and more interdependent, and understanding how robustness is affected by these interdependences is one of the main challenges faced when designing resilient infrastructures ${ }^{67,69-72}$. In recent decades, research was carried out in applied science on cataloguing, analysing and modelling the interdependences in critical infrastructure as well as modelling cascading failures in coupled critical infrastructure networks ${ }^{40,42-48}$. However, no systematic mathematical framework, such as percolation theory, is currently available for adequately addressing the consequences of disruptions and failures occurring simultaneously in interdependent critical infrastructures.

Recently, motivated by the fact that modern, crucially important infrastructures significantly interact, a mathematical framework was developed ${ }^{73}$ to study percolation in a system of two interdependent networks subject to cascading failure. The analytical framework is based on a generating-function formalism widely used for studies of percolation and structure within a single network ${ }^{73-75}$. The framework for interdependent networks enables us to follow the dynamics of the cascading failures as well as to derive the analytic solutions for the final steady state. It was found ${ }^{73}$ that certain types of interdependent network were significantly more vulnerable than their non-interacting counterparts. The failure of even a small number of elements within a single network may trigger a catastrophic cascade of events that destroys the global connectivity. For a fully interdependent case in which each node in one network depends on a functioning node in other networks and vice versa, a first-order discontinuous phase transition, which is dramatically different from the second-order continuous phase transition found in isolated networks (Fig. 1), was found ${ }^{73}$. This phenomenon is caused by the presence of two types of link: connectivity links within each network; and dependence links between networks. Connectivity links enable the network to carry out its function and dependence links represent the fact that the function of a given node in one network depends crucially on nodes in other networks. The case of connectivity links between the different networks was studied in ref. 66. It was shown ${ }^{76}$ that, when the dependence coupling between the networks is reduced, at a critical coupling strength the percolation transition becomes second order.

More recently, two important generalizations of the basic model of ref. 73 have been developed:
One generalization takes into account that in real-world scenarios the initial failure of important nodes (or hubs) may be not random but targeted. A mathematical framework for understanding the robustness of interdependent networks under an initial targeted attack has been studied in ref. 77 . The authors of that work developed a general technique that uses the random-attack problem to map the targeted-attack problem in interdependent networks.

The other generalization takes into account that, in real-world scenarios, the assumption that each node in network A depends on one and only one node in network B and vice versa may not be valid. To correct this shortcoming, a theoretical framework for understanding the robustness of interdependent networks with a random number of support and dependence relationships has been developed and studied ${ }^{78}$.

In all of the above studies ${ }^{73,76-78}$, the dependent pairs of nodes in both networks were chosen randomly. Thus when highdegree nodes in one network depend with a high probability on low-degree nodes of another network the configuration becomes vulnerable. To quantify and better understand this phenomenon, we proposed two 'intersimilarity' measures between the interdependent networks ${ }^{79}$. On the one hand, intersimilarity occurs in interdependent networks when nodes with similar degrees tend to be interdependent. On the other hand, it occurs if the neighbours of interdependent nodes in each network also tend to be interdependent. Refs $79-81$ found that as the interdependent networks become more intersimilar the system becomes more robust. A system composed of an interdependent world-wide seaport and airport networks and the world-wide airport network was studied in ref. 79, where it was found that well-connected seaports tend to couple with well-connected airports, and two ways of measuring the intersimilarity of interdependent networks were developed. The case in which all pairs of interdependent nodes in both networks have the same degree was solved analytically in ref. 82 .

The robustness of a two-coupled-networks system has been studied for dependence coupling ${ }^{73}$ and for connectivity coupling ${ }^{66}$. Very recently a more realistic coupled network system with both dependence and connectivity links between the coupled networks was studied ${ }^{83}$. Using a percolation approach, rich and unusual phase transition phenomena were found, including a mixed first-order and second-order hybrid transition. This hybrid transition shows that a discontinuous jump in the size of the giant component (as in a first-order transition) is followed by a continuous decrease to zero (as in a second-order transition).

Previous studies of isolated networks in which dependence links cause cascading failure fall into two categories:

The first studies failures due to network overload when the network flow is a physical quantity, for example, in power transmission systems, transportation networks, or Internet traffic ${ }^{84-87}$. The models produced by these studies demonstrate that when an overloaded node stops traffic flow, the choosing of alternative paths can overload other nodes, and a cascading failure that disables the entire network can result.

The second is studies that produce models based on local dependences, such as the decision-making of interacting agents ${ }^{11}$. In these models the state of a node depends on the state of its neighbours, that is, a failing node will cause its neighbours to also fail.

The rich phenomena found in interdependent networks and the insights obtained from the percolation framework developed in refs 73,76 have led to a better understanding of the effect of dependence links within single isolated networks. A percolation approach for a single network in the presence of random dependence links was developed recently ${ }^{88-90}$. The results show that cascading failures occur, yielding a first-order transition, and that 
the percolation threshold of the network significantly increases with an increase in the number of dependence links.

\section{Generating functions for a single network}

We begin by describing the generating-function formalism ${ }^{74}$ for a single network that will also be useful in studying interdependent networks. We assume that all $N_{i}$ nodes in network $i$ are randomly assigned a degree $k$ from a probability distribution $P_{i}(k)$, and are randomly connected with the only constraint that the node with degree $k$ has exactly $k$ links ${ }^{91}$. We define the generating function of the degree distribution

$$
G_{i}(x) \equiv \sum_{k=0}^{\infty} P_{i}(k) x^{k}
$$

where $x$ is an arbitrary complex variable. Using equation (1), the average degree of network $i$ is

$$
\langle k\rangle_{i}=\sum_{k=0}^{\infty} k P_{i}(k)=\left.\frac{\partial G_{i}}{\partial x}\right|_{x \rightarrow 1}=G_{i}^{\prime}(1)
$$

In the limit of infinitely large networks $N_{i} \rightarrow \infty$, the random connection process can be modelled as a branching process in which an outgoing link of any node has a probability $k P_{i}(k) /\langle k\rangle_{i}$ of being connected to a node with degree $k$, which in turn has $k-1$ outgoing links. Using equations (1) and (2), the generating function of this branching process is defined as

$$
H_{i}(x) \equiv \frac{\sum_{k=0}^{\infty} P_{i}(k) k x^{k-1}}{\langle k\rangle_{i}}=\frac{G_{i}^{\prime}(x)}{G_{i}^{\prime}(1)}
$$

Let $f_{i}$ be the probability that a randomly selected link does not lead to the giant component. If a link leads to a node with $k-1$ outgoing links this probability is $f_{i}^{k-1}$. Thus $H_{i}\left(f_{i}\right)$ also has the meaning that a randomly selected link does not lead to the giant component and hence $f_{i}$ satisfies the recursive relation equation $f_{i}=H_{i}\left(f_{i}\right)$. The probability that a node with degree $k$ does not belong to the giant component is $f_{i}^{k}$ and hence the probability that a randomly selected node belongs to the giant component is $g_{i}=1-G_{i}\left(f_{i}\right)$.

Once a fraction $1-p$ of nodes is randomly removed from a network, the generating function remains the same, but with a new argument $z_{i} \equiv p x+1-p$ (ref. 75). Accordingly, owing to the definition of $f_{i}$ and $g_{i}$ the probability that a randomly chosen surviving node belongs to a giant component is given by

$$
g_{i}(p)=1-G_{i}\left[p f_{i}(p)+1-p\right]
$$

where $f_{i}(p)$ satisfies

$$
f_{i}(p)=H_{i}\left[p f_{i}(p)+1-p\right]
$$

Thus $P_{\infty, i}$, the fraction of nodes that belongs to the giant component, is given by the product ${ }^{75}$

$$
P_{\infty, i}=p g_{i}(p)
$$

As $p$ decreases, the non-trivial solution $f_{i}<1$ of equation (5) gradually approaches the trivial solution $f_{i}=1$. Accordingly, $P_{\infty, i}$ gradually approaches zero as in a second-order phase transition and becomes zero when two solutions of equation (5) coincide at $p=p_{\mathrm{c}}$. At this point the straight line corresponding to the left-hand side of equation (5) becomes tangent to the curve corresponding to its right-hand side, yielding

$$
p_{\mathrm{c}}=1 / H^{\prime}{ }_{i}(1)
$$

For example, for Erdős-Rényi networks ${ }^{92-94}$, characterized by a Poisson degree distribution, using equations (1), (3) and (7) we obtain

$$
\begin{gathered}
G_{i}(x)=H_{i}(x)=\exp \left[\langle k\rangle_{i}(x-1)\right] \\
g_{i}(p)=1-f_{i}(p) \\
f_{i}(p)=\exp \left\{p\langle k\rangle_{i}\left[f_{i}(p)-1\right]\right\}
\end{gathered}
$$

and using equations (7) and (8)

$$
p_{\mathrm{c}}=\frac{1}{\langle k\rangle_{i}}
$$

Finally, using equations (6), (9) and (10), we obtain a direct equation for $P_{\infty, i}$

$$
P_{\infty, i}=p\left[1-\exp \left(-\langle k\rangle_{i} P_{\infty, i}\right)\right]
$$

\section{Framework of two partially interdependent networks}

A generalization of the percolation theory of two fully interdependent networks ${ }^{73}$ has been developed by Parshani et al. ${ }^{76}$, where a more realistic case of a pair of partially interdependent networks has been studied. In this case, both interacting networks have a certain fraction of completely autonomous nodes whose function does not directly depend on the nodes of the other network. It has been found that, once the fraction of autonomous nodes increases above a certain threshold, the abrupt collapse of the interdependent networks characterized by a first-order transition observed in ref. 73 changes, at a critical coupling strength, to a continuous secondorder transition as in classical percolation theory ${ }^{52}$.

In the following we describe in more detail the framework developed in ref. 76. This framework consists of two networks A and B with the numbers of nodes $N_{\mathrm{A}}$ and $N_{\mathrm{B}}$, respectively. Within network $\mathrm{A}$, the nodes are randomly connected by $\mathrm{A}$ edges with degree distribution $P_{\mathrm{A}}(k)$, whereas the nodes in network $\mathrm{B}$ are randomly connected by $\mathrm{B}$ edges with degree distribution $P_{\mathrm{B}}(k)$. The average degrees of the networks $\mathrm{A}$ and $\mathrm{B}$ are $a$ and $b$ respectively. In addition, a fraction $q_{\mathrm{A}}$ of network A nodes depends on the nodes in network $\mathrm{B}$ and a fraction $q_{\mathrm{B}}$ of network $\mathrm{B}$ nodes depends on the nodes in network $\mathrm{A}$. We assume that a node from one network depends on no more than one node from the other network, and if node $\mathrm{A}_{i}$ depends on node $\mathrm{B}_{j}$, and $\mathrm{B}_{j}$ depends on $\mathrm{A}_{k}$, then $k=i$. The latter condition, which we call a no-feedback condition (Fig. 2), excludes configurations that completely collapse even for fully interdependent networks once a single node is removed ${ }^{78}$. We assume that the initial removal of nodes from network A is a fraction $1-p$.

Next we present the formalism for the cascade process step by step (Fig. 3). After an initial removal of nodes, the remaining fraction of nodes in network $\mathrm{A}$ is $\psi_{1}^{\prime} \equiv p$. The initial removal of nodes will disconnect some nodes from the giant component. The remaining functional part of network A therefore constitutes a fraction $\psi_{1}=\psi_{1}^{\prime} g_{\mathrm{A}}\left(\psi_{1}^{\prime}\right)$ of the network nodes, where $g_{\mathrm{A}}\left(\psi_{1}^{\prime}\right)$ is defined by equations (4) and (5). As a fraction $q_{\mathrm{B}}$ of nodes from network $B$ depends on nodes from network $A$, the number of nodes in network $B$ that become non-functional is 
a

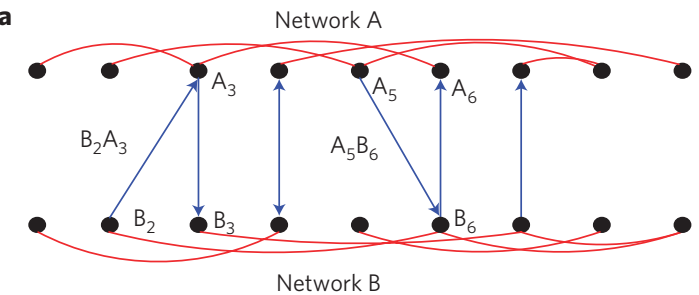

b

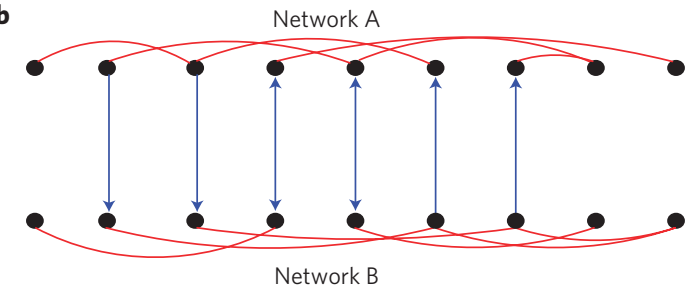

Figure 2 | Differences between the feedback condition and no-feedback condition. $\mathbf{a}, \mathbf{b}$ In the case of feedback (a), node $A_{3}$ depends on node $B_{2}$, and node $B_{3} \neq B_{2}$ depends on node $A_{3}$, whereas if there is no feedback (b) this is forbidden. The blue links between the two networks show the dependence links and the red links in each network show the connectivity links, which enable each network to function.

$\left(1-\psi_{1}\right) q_{\mathrm{B}}=q_{\mathrm{B}}\left[1-\psi_{1}^{\prime} g_{\mathrm{A}}\left(\psi_{1}^{\prime}\right)\right]$. Accordingly, the remaining fraction of network B nodes is $\phi_{1}^{\prime}=1-q_{\mathrm{B}}\left[1-\psi_{1}^{\prime} g_{\mathrm{A}}\left(\psi_{1}^{\prime}\right)\right]$, and the fraction of nodes in the giant component of network $\mathrm{B}$ is $\phi_{1}=\phi_{1}^{\prime} g_{\mathrm{B}}\left(\phi_{1}^{\prime}\right)$.

Following this approach we can construct the sequence, $\psi_{t}^{\prime}$ and $\phi_{t}^{\prime}$, of the remaining fraction of nodes at each stage of the cascade of failures. The general form is given by

$$
\begin{aligned}
& \psi_{1}^{\prime} \equiv p \\
& \phi_{1}^{\prime}=1-q_{\mathrm{B}}\left[1-p g_{\mathrm{A}}\left(\psi_{1}^{\prime}\right)\right] \\
& \psi_{t}^{\prime}=p\left[1-q_{\mathrm{A}}\left(1-g_{\mathrm{B}}\left(\phi_{t-1}^{\prime}\right)\right)\right] \\
& \phi_{t}^{\prime}=1-q_{\mathrm{B}}\left[1-p g_{\mathrm{A}}\left(\psi_{t-1}^{\prime}\right)\right]
\end{aligned}
$$

To determine the state of the system at the end of the cascade process we look at $\psi_{\tau}^{\prime}$ and $\phi_{\tau}^{\prime}$ at the limit of $\tau \rightarrow \infty$. This limit must satisfy the equations $\psi_{\tau}^{\prime}=\psi_{\tau+1}^{\prime}$ and $\phi_{\tau}^{\prime}=\phi_{\tau+1}^{\prime}$ because eventually the clusters stop fragmenting and the fractions of randomly removed nodes at steps $\tau$ and $\tau+1$ are equal. Denoting $\psi_{\tau}^{\prime}=x$ and $\phi_{\tau}^{\prime}=y$, we arrive in the stationary state at a system of two equations with two unknowns,

$$
\begin{aligned}
& x=p\left\{1-q_{\mathrm{A}}\left[1-g_{\mathrm{B}}(y)\right]\right\} \\
& y=1-q_{\mathrm{B}}\left[1-g_{\mathrm{A}}(x) p\right]
\end{aligned}
$$

The giant components of networks $\mathrm{A}$ and $\mathrm{B}$ at the end of the cascade of failures are, respectively, $P_{\infty, A}=\psi_{\infty}=x g_{\mathrm{A}}(x)$ and $P_{\infty, B}=\phi_{\infty}=y g_{\mathrm{B}}(y)$. Figure 4 shows the excellent agreement for the cascading failures in the giant component between computer simulations and the analytical results. The analytical results were obtained by recursive relations (13), where $g_{\mathrm{A}}\left(\psi_{t}^{\prime}\right)$ and $g_{\mathrm{B}}\left(\phi_{t}^{\prime}\right)$ are computed using equations (9) and (10).

Equation (14) can be illustrated graphically by two curves crossing in the $(x, y)$ plane. For sufficiently large $q_{\mathrm{A}}$ and $q_{\mathrm{B}}$ the curves intersect at two points $\left(0<x_{0}, 0<y_{0}\right)$ and $\left(x_{0}<x_{1}<1, y_{0}<y_{1}<1\right)$. Only the second solution $\left(x_{1}, y_{1}\right)$ has a physical meaning. As $p$ decreases, the two solutions become closer to each other, remaining inside the unit square $(0<x<1 ; 0<y<1)$, and at a certain threshold $p=p_{\mathrm{c}}$ they coincide: $0<x_{0}=x_{1}=x_{\mathrm{c}}<1,0<y_{0}=y_{1}=y_{\mathrm{c}}<1$. a

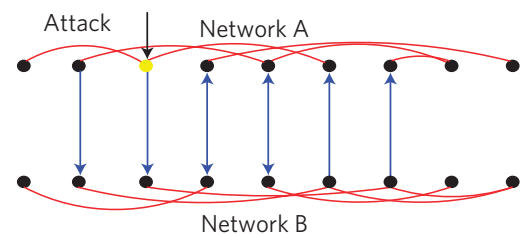

b.

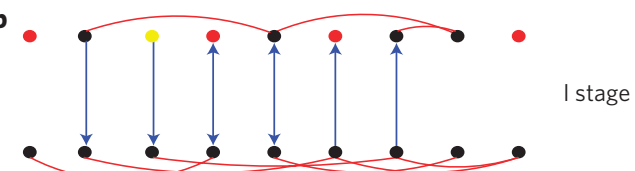

c
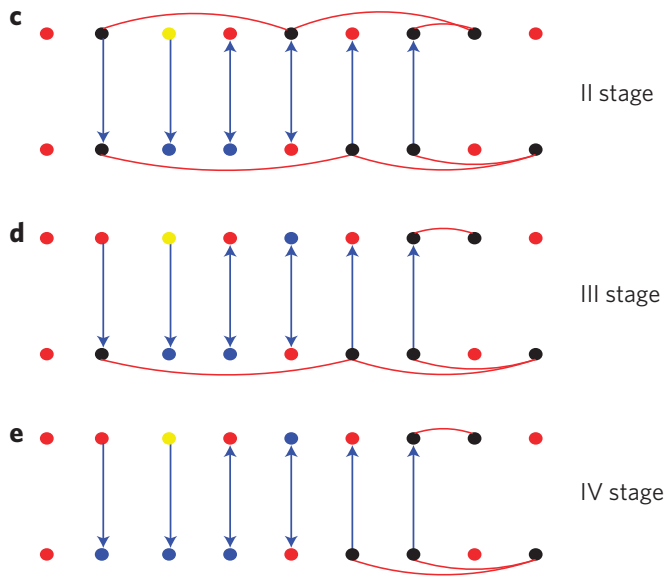

Figure 3 | Description of the dynamic process of cascading failures on two partially interdependent networks, which can be generalized to $n$ partially interdependent networks. The black nodes represent the survival nodes, the yellow node represents the initially attacked node, the red nodes represent the nodes removed because they do not belong to the largest cluster and the blue nodes represent the nodes removed because they depend on the failed nodes in the other network. In each stage, for one network, we first remove the nodes that depend on the failed nodes in the other network or on the initially attacked nodes. Next we remove the nodes that do not belong to the largest cluster of the network.

For $p<p_{\mathrm{c}}$ the non-trivial solution corresponding to the intersection abruptly disappears. Thus for sufficiently large $q_{\mathrm{A}}$ and $q_{\mathrm{B}}, P_{\infty, A}$ and $P_{\infty, B}$ as a function of $p$ show a first-order phase transition. As $q_{\mathrm{B}}$ decreases, the intersection of the curves moves out of the unit square; therefore, for small enough $q_{\mathrm{B}}, P_{\infty, A}$ as a function of $p$ shows a second-order phase transition. For the graphical representation of equation (14) and all possible solutions see Fig. 3 in ref. 76.

In a recent study ${ }^{95}$, it was shown that a pair of interdependent networks can be designed to be more robust by choosing the autonomous nodes to be high-degree nodes. This choice mitigates the probability of catastrophic cascading failure.

\section{Framework for a network of interdependent networks}

In many real systems there are more than two interdependent networks, and diverse infrastructures - water and food supply networks, communication networks, fuel networks, financial transaction networks or power-station networks - can be coupled together ${ }^{69,70}$. Understanding the way system robustness is affected by such interdependences is one of the main challenges when designing resilient infrastructures.

Here we review the generalization of the theory of a pair of interdependent networks ${ }^{73,76}$ to a system of $n$ interacting networks ${ }^{96}$, which can be graphically represented (Fig. 5) as a network of networks (NON). We develop an exact analytical 


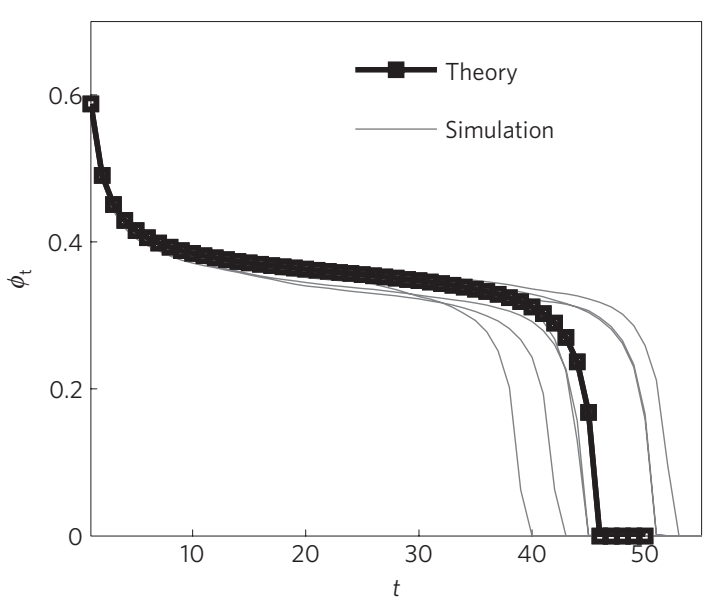

Figure 4 | Cascade of failures in two partially interdependent Erdős-Rényi networks. The giant component $\phi_{t}$ for every iteration of the cascading failures is shown for the case of a first-order phase transition with the initial parameters $p=0.8505, a=b=2.5, q_{\mathrm{A}}=0.7$ and $q_{\mathrm{B}}=0.8$. In the simulations, $N=2 \times 10^{5}$ with over 20 realizations. The grey lines represent different realizations. The squares represent the average over all realizations and the black line is obtained from equation (13).

approach for percolation of an NON system composed of $n$ fully or partially interdependent randomly connected networks. The approach is based on analysing the dynamical process of the cascading failures. The results generalize the known results for percolation of a single network $(n=1)$ and the $n=2$ result found in refs 73,76, and show that, whereas for $n=1$ the percolation transition is a second-order transition, for $n>1$ cascading failures occur and the transition becomes first order. Our results for $n$ interdependent networks suggest that the classical percolation theory extensively studied in physics and mathematics is a limiting case of $n=1$ of a general theory of percolation in NON. As we shall discuss here this general theory has many features that are not present in the classical percolation theory.

In our generalization, each node in the NON is a network itself and each link represents a fully or partially dependent pair of networks. We assume that each network $i(i=1,2, \ldots, n)$ of the NON consists of $N_{i}$ nodes linked together by connectivity links. Two networks $i$ and $j$ form a partially dependent pair if a certain fraction $q_{j i}>0$ of nodes of network $i$ directly depends on nodes of network $j$, that is, they cannot function if the nodes in network $j$ on which they depend do not function. Dependent pairs are connected by unidirectional dependence links pointing from network $j$ to network $i$. This convention symbolizes the fact that nodes in network $i$ receive supply from nodes in network $j$ of a crucial commodity, for example electric power if network $j$ is a power grid.

We assume that after an attack or failure only a fraction of nodes $p_{i}$ in each network $i$ will remain. We also assume that only nodes that belong to a giant connected component of each network $i$ will remain functional. This assumption helps explain the cascade of failures: nodes in network $i$ that do not belong to its giant component fail, causing failures of nodes in other networks that depend on the failing nodes of network $i$. The failure of these nodes causes the direct failure of the dependent nodes in other networks, failures of isolated nodes in them and further failure of nodes in network $i$, and so on. Our goal is to find the fraction of nodes $P_{\infty, i}$ of each network that remain functional at the end of the cascade of failures as a function of all fractions $p_{i}$ and all fractions $q_{i j}$. We assume that all networks in the NON are randomly connected networks characterized by a degree distribution of links $P_{i}(k)$, where $k$ is a degree of a node in network $i$. We further assume that each

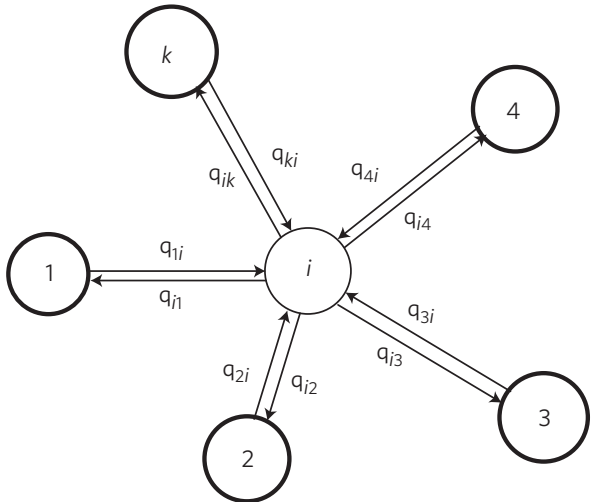

Figure 5 | Schematic representation of a NON. Circles represent interdependent networks, and the arrows connect the partially interdependent pairs. For example, a fraction of $q_{3 i}$ of nodes in network $i$ depend on the nodes in network 3. The networks that are not connected by the dependence links do not have nodes that directly depend on one another.

node $a$ in network $i$ may depend with probability $q_{j i}$ on only one node $b$ in network $j$.

We can study different models of cascading failures in which we vary the survival time of the dependent nodes after the failure of the nodes in other networks on which they depend and the survival time of the disconnected nodes. We conclude that the final state of the networks does not depend on these details but can be described by a system of equations somewhat analogous to the Kirchhoff equations for a resistor network. This system of equations has $n$ unknowns $x_{i}$. These represent the fractions of nodes that survive in network $i$ after the nodes that fail in the initial attack are removed, and also the nodes depending on the failed nodes in other networks at the end of cascading failure are removed, but without considering yet the further failing of nodes due to the internal connectivity of the network. The final giant component of each network can be found from the equation $P_{\infty, i}=x_{i} g_{i}\left(x_{i}\right)$, where $g_{i}\left(x_{i}\right)$ is the fraction of the remaining nodes of network $i$ that belong to its giant component given by equation (4).

First we shall discuss the more complex case of the no-feedback condition. The unknowns $x_{i}$ satisfy the system of $n$ equations,

$$
x_{i}=p_{i} \prod_{j=1}^{K}\left[q_{j i} y_{j i} g_{j}\left(x_{j}\right)-q_{j i}+1\right]
$$

where the product is taken over the $K$ networks interlinked with network $i$ by the partial dependence links (Fig. 3) and

$$
y_{i j}=\frac{x_{i}}{q_{j i} y_{j i} g_{j}\left(x_{j}\right)-q_{j i}+1}
$$

has the meaning of the fraction of nodes in network $j$ that survive after the damage from all the networks connected to network $j$ except network $i$ is taken into account. The damage from network $i$ must be excluded owing to the no-feedback condition. In the absence of the no-feedback condition, equation (15) becomes much simpler as $y_{j i}=x_{j}$. Equation (15) is valid for any case of interdependent NON, whereas equation (16) represents the no-feedback condition.

\section{Four examples of a NON solvable analytically}

In this section we present four examples that can be explicitly solved analytically: (1) a tree-like Erdős-Rényi fully dependent 


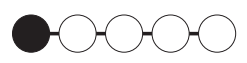

Chain-like NON

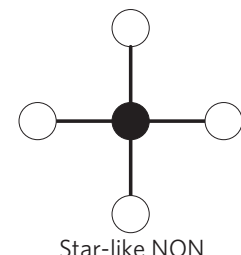

Star-like NON

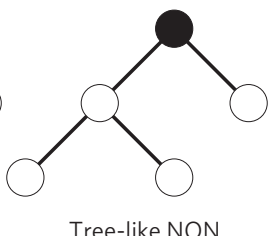

Tree-like NON
Figure 6 | Three types of loopless NON composed of five coupled networks. All have the same percolation threshold and the same giant component. The dark node represents the origin network on which failures initially occur.

NON, (2) a tree-like random regular fully dependent NON, (3) a loop-like Erdős-Rényi partially dependent NON and (4) a random regular network of partially dependent Erdős-Rényi networks. All cases represent different generalizations of percolation theory for a single network. In all examples except (3) we apply the no-feedback condition.

(1) We solve explicitly ${ }^{96}$ the case of a tree-like NON (Fig. 6) formed by $n$ Erdôs-Rényi networks ${ }^{92-94}$ with the same average degrees $k, p_{1}=p, p_{i}=1$ for $i \neq 1$ and $q_{i j}=1$ (fully interdependent). From equations (15) and (16) we obtain an exact expression for the order parameter, the size of the mutual giant component for all $p, k$ and $n$ values,

$$
P_{\infty}=p\left[1-\exp \left(-k P_{\infty}\right)\right]^{n}
$$

Equation (17) generalizes known results for $n=1,2$. For $n=1$, we obtain the known result $p_{\mathrm{c}}=1 / k$, equation (11), of an Erdős-Rényi network and $P_{\infty}\left(p_{c}\right)=0$, which corresponds to a continuous second-order phase transition. Substituting $n=2$ in equation (17) yields the exact results of ref. 73 .

Solutions of equation (17) are shown in Fig. 7a for several values of $n$. The special case $n=1$ is the known Erdös-Rényi second-order percolation law, equation (12), for a single network. In contrast, for any $n>1$, the solution of (17) yields a first-order percolation transition, that is, a discontinuity of $P_{\infty}$ at $p_{c}$.

Our results show (Fig. 7a) that the NON becomes more vulnerable with increasing $n$ or decreasing $k$ ( $p_{\mathrm{c}}$ increases when $n$ increases or $k$ decreases). Furthermore, for a fixed $n$, when $k$ is smaller than a critical number $k_{\min }(n), p_{\mathrm{c}} \geq 1$, meaning that for $k<k_{\min }(n)$ the NON will collapse even if a single node fails ${ }^{96}$.

(2) In the case of a tree-like network of interdependent random regular networks ${ }^{97}$, where the degree $k$ of each node in each network is assumed to be the same, we obtain an exact expression for the order parameter, the size of the mutual giant component for all $p, k$ and $n$ values,

$$
P_{\infty}=p\left\{1-\left\{p^{\frac{1}{n}} P_{\infty}^{\frac{n-1}{n}}\left[\left(1-\left(\frac{P_{\infty}}{p}\right)^{\frac{1}{n}}\right)^{\frac{k-1}{k}}-1\right]+1\right\}^{k}\right\}^{n}
$$

Numerical solutions of equation (18) are in excellent agreement with simulations. Comparing with the results of the tree-like Erdös-Rényi NON, we find that the robustness of $n$ interdependent random regular networks of degree $k$ is significantly higher than that of the $n$ interdependent Erdös-Rényi networks of average degree $k$. Moreover, whereas for an Erdős-Rényi NON there exists a critical minimum average degree $k=k_{\min }$ that increases with $n$ (below which the system collapses), there is no such analogous $k_{\min }$ for the random regular NON system. For any $k>2$, the random regular NON is stable, that is, $p_{\mathrm{c}}<1$. In general, this is correct for any network with any degree distribution, $P_{i}(k)$, such that
$P_{i}(0)=P_{i}(1)=0$, that is, for a network without disconnected or singly connected nodes ${ }^{97}$.

(3) In the case of a loop-like NON (for dependences in one direction) of $n$ Erdös-Rényi networks ${ }^{96}$, all the links are unidirectional, and the no-feedback condition is irrelevant. If the initial attack on each network is the same, $1-p, q_{i-1 i}=q_{n 1}=q$ and $k_{i}=k$, using equations (15) and (16) we obtain that $P_{\infty}$ satisfies

$$
P_{\infty}=p\left(1-\mathrm{e}^{-k P_{\infty}}\right)\left(q P_{\infty}-q+1\right)
$$

Note that if $q=1$ equation (19) has only a trivial solution $P_{\infty}=0$, whereas for $q=0$ it yields the known giant component of a single network, equation (12), as expected. We present numerical solutions of equation (19) for two values of $q$ in Fig. 7b. Interestingly, whereas for $q=1$ and tree-like structures equations (17) and (18) depend on $n$, for loop-like NON structures equation (19) is independent of $n$.

(4) For NONs where each ER network is dependent on exactly $m$ other Erdős-Rényi networks (the case of a random regular network of Erdôs-Rényi networks), we assume that the initial attack on each network is $1-p$, and each partially dependent pair has the same $q$ in both directions. The $n$ equations of equation (15) are exactly the same owing to symmetries, and hence $P_{\infty}$ can be obtained analytically,

$$
P_{\infty}=\frac{p}{2^{m}}\left(1-\mathrm{e}^{-k P_{\infty}}\right)\left[1-q+\sqrt{(1-q)^{2}+4 q P_{\infty}}\right]^{m}
$$

from which we obtain

$$
p_{\mathrm{c}}=\frac{1}{k(1-q)^{m}}
$$

Again, as in case (3), it is surprising that both the critical threshold and the giant component are independent of the number of networks $n$, in contrast to tree-like NON (equations (17) and (18)), but depend on the coupling $q$ and on both degrees $k$ and $m$. Numerical solutions of equation (20) are shown in Fig. 7c, and the critical thresholds $p_{c}$ in Fig. $7 c$ coincide with the theory, equation (21).

\section{Remark on scale-free networks}

The above examples regarding Erdós-Rényi and random regular networks have been selected because they can be explicitly solved analytically. In principle, the generating function formalism presented here can be applied to randomly connected networks with any degree distribution. The analysis of the scale-free networks with a power-law degree distribution $P(k) \sim k^{-\lambda}$ is extremely important, because many real networks can be approximated by a power-law degree distribution, such as the Internet, the airline network and social-contact networks, such as networks of scientific collaboration ${ }^{2,10,51}$. Analysis of fully interdependent scale-free networks ${ }^{73}$ shows that, for interdependent scale-free networks, $p_{\mathrm{c}}>0$ even in the case $\lambda \leq 3$ for which in a single network $p_{\mathrm{c}}=0$. In general, for fully interdependent networks, the broader the degree distribution the greater $p_{c}$ for networks with the same average degree ${ }^{73}$. This means that networks with a broad degree distribution become less robust than networks with a narrow degree distribution. This trend is the opposite of the trend found in non-interacting isolated networks. The explanation of this phenomenon is related to the fact that in randomly interdependent networks the hubs in one network may depend on poorly connected nodes in another. Thus the removal of a randomly selected node in one network may cause a failure of a hub in a second network, which in turn renders many singly connected 

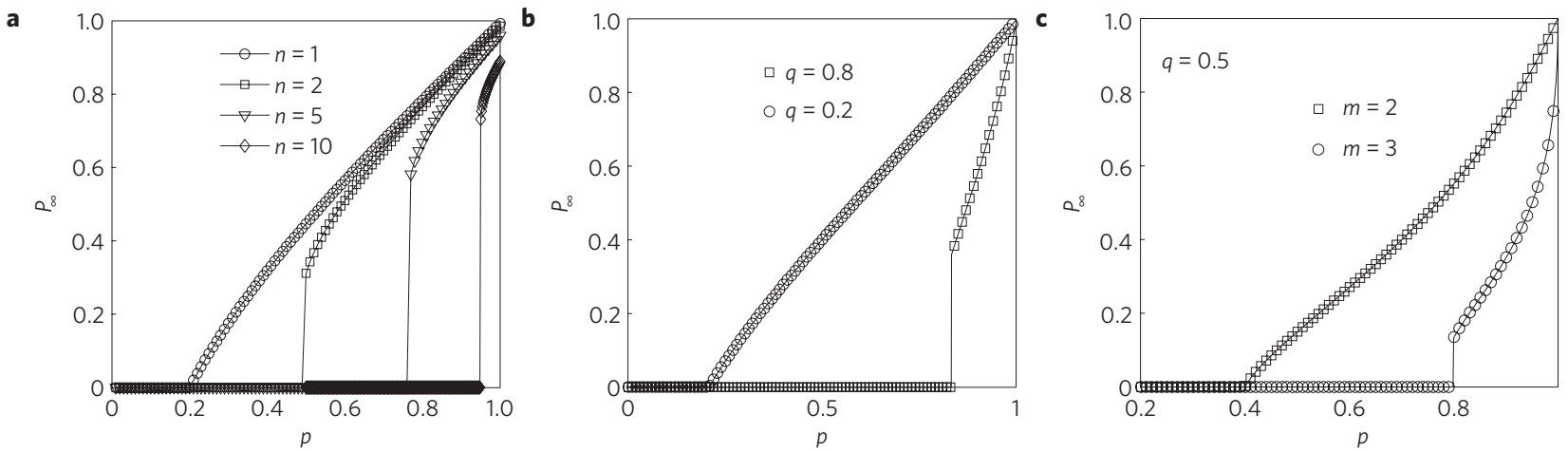

Figure 7 | The fraction of nodes in the giant component $\boldsymbol{P}_{\infty}$ as a function of $\boldsymbol{p}$ for three different examples. $\mathbf{a}, \mathrm{A}$ tree-like fully ( $q=1$ ) interdependent NON; $P_{\infty}$ is shown as a function of $p$ for $k=5$ and several values of $n$. The results are obtained using equation (17). Note that increasing $n$ from $n=2$ yields a first-order transition. $\mathbf{b}$, A loop-like NON; $P_{\infty}$ is shown as a function of $p$ for $k=6$ and two values of $q$. The results are obtained using equation (19). Note that increasing $q$ yields a first-order transition. $\mathbf{c}$, A random regular network of Erdős-Rényi networks; $P_{\infty}$ is shown as a function of $p$, for two different values of $m$ when $q=0.5$. The results are obtained using equation (20), and the number of networks, $n$, can be any number with the condition that any network in the NON connects exactly to $m$ other networks. Note that changing $m$ from 2 to $m>2$ changes the transition from second order to first order (for $q=0.5$ ).

nodes non-functional, and the multiplying damage travels back to the first network. This explanation is corroborated by the analytical proof in ref. 82, which shows that if the degrees of the interdependent nodes coincide, then a network with a broader degree distribution will become more robust than a network with a narrower degree distribution, that is, the behaviour characteristic of non-interacting networks is restored. Ref. 82 also reports that, for fully interdependent scale-free networks with equal degrees of interdependent pairs, $p_{\mathrm{c}}=0$ for $\lambda<3$. Moreover, the percolation transition is a discontinuous first-order phase transition if and only if $H_{i}^{\prime}(1)<\infty$, that is, if the degree distribution has a finite second moment. For fully interdependent networks with uncorrelated degrees of interdependent nodes, the percolation transition is always a discontinuous phase transition ${ }^{73,76}$. These results, as well as the results of ref. 79, show the need to study more realistic situations in which the interdependent networks have various correlations in the dependences and connectivities. A recent study of partially interdependent scale-free networks shows that, although the giant component decreases significantly owing to cascading failures, $p_{\mathrm{c}}$ is always zero as long as $q<1$ (D. Zhou et al., unpublished).

\section{Remaining challenges}

We have reviewed recent studies of the robustness of a system of interdependent networks. In interacting networks, when a node in one network fails it usually causes dependent nodes in other networks to fail, which, in turn, may cause further damage in the first network and results in a cascade of failures with catastrophic consequences. Our analytical framework enables us to follow the dynamic process of the cascading failures step by step and to derive steady-state solutions. Interdependent networks appear in all aspects of life, nature and technology. Transportation systems include railway networks, airline networks and other transportation systems. Some properties of interacting transportation systems have been studied recently ${ }^{79,80}$. In the field of physiology, the human body can be regarded as a system of interdependent networks. Examples of such interdependent NON systems include the cardiovascular system, the respiratory system, the brain neuron system and the nervous system. In biology, the function of each protein is determined by its interacting proteins, which can be described by a network. As many proteins are involved in a number of different functions, the protein-interaction system can be regarded as a system of interacting networks. In the field of economics, networks of banks, insurance companies and business firms are interdependent.
Thus far, only a very few real-world interdependent systems have been analysed using the percolation approach ${ }^{71,79,80}$. We expect our present work to provide insights leading to a further analysis of real data on interdependent networks. The benchmark models we present here can be used to study the structural, functional and robustness properties of interdependent networks. Because, in real NONs, individual networks are not randomly connected and their interdependent nodes are not selected at random, it is crucial that we understand the many types of correlation that exist in real-world systems and that we further develop the theoretical tools to include such correlations. Further studies of interdependent networks should focus on an analysis of real data from many different interdependent systems and on the development of mathematical tools for studying real-world interdependent systems.

Many real-world networks are embedded in space, and the spatial constraints strongly affect their properties ${ }^{30}$. We need to understand how these spatial constraints influence the robustness properties of interdependent networks ${ }^{79,80}$. Other properties that influence the robustness of single networks, such as the dynamic nature of the configuration in which links or nodes appear and disappear and the directed nature of some links, as well as problems associated with degree-degree correlations and clustering, should be also addressed in future studies of coupled network systems. It is also important to investigate the case when a node in one network is supplied by multiple nodes in an interdependent network. In realistic interdependent pairs of networks $i$ and $j$, a node in network $i$ may depend on $s$ supply nodes in network $j$ and the total supply of a commodity received by this node from network $j$ must be greater than a certain threshold $s_{\mathrm{c}}$. In the case of $s_{\mathrm{c}}=0$ and random selection of the supply nodes, this problem was solved in ref. 78 for two interdependent networks, and this solution can be straightforwardly generalized for an arbitrary NON by replacing equation (15) with

$$
x_{i}=p_{i} \prod_{j=1}^{K}\left\{1-q_{j i} G^{j i}\left[1-x_{j} g_{j}\left(x_{j}\right)\right]\right\}
$$

where $G^{j i}(x)$ is the generating function of the distribution of the supply degree $s$ of nodes in network $i$ that depend on the supply from nodes in network $j$. When $s=1$ for all such nodes, $G^{j i}(x)=x$ and equation (22) reduces to equation (15) with $y_{j i}=x_{j}$, that is, in the absence of the no-feedback condition. More complex cases of multiple supply nodes await further investigation.

It is very important to find a way of improving the robustness of interdependent infrastructures. Our studies thus far show that 
there are three methods to achieve this goal: increase the fraction of autonomous nodes ${ }^{76}$, particularly nodes with high degree ${ }^{95}$; design the dependence links such that they connect the nodes with similar degrees ${ }^{79,82}$ and protect the high-degree nodes against attack ${ }^{95}$.

A coupled network in which the interlinks, that is, the links between different networks, are connectivity links was studied in ref. 66. The robustness of this system is greatly improved when compared with a system in which the interlinks are dependence links. A systematic study of the competing effects of a NON in which the interlinks are both dependence and connectivity interlinks is needed. Interesting results on a model containing both dependence and connectivity interlinks have been obtained ${ }^{83}$. Finally, we mention an early study of the Ising model on coupled networks ${ }^{98}$. Also, interacting networks with respect to climate systems were studied in ref. 99 .

\section{References}

1. Watts, D. J. \& Strogatz, S. H. Collective dynamics of 'small-world' networks. Nature 393, 440-442 (1998).

2. Barabási, A. L. \& Albert, R. Emergence of scaling in random networks. Science 286, 509-512 (1999).

3. Faloutsos, M., Faloutsos, P. \& Faloutsos, C. On power-law relationships of the internet topology. Comput. Commun. Rev. 29, 378-382 (2000).

4. Albert, R., Jeong, H. \& Barabási, A. L. Error and attack tolerance of complex networks. Nature 406, 378-382 (2000).

5. Cohen, R., Erez, K., Ben-Avraham, D. \& Havlin, S. Resilience of the Internet to random breakdown. Phys. Rev. Lett. 85, 4626-4628 (2000)

6. Callaway, D. S., Newman, M. E. J., Strogatz, S. H. \& Watts, D. J. Network robustness and fragility: Percolation on random graphs. Phys. Rev. Lett. 85, 5468-5471 (2000).

7. Cohen, R., Erez, K., Ben-Avraham, D. \& Havlin, S. Breakdown of the Internet under intentional attack. Phys. Rev. Lett. 86, 3682-3685 (2001).

8. Strogatz, S. H. Exploring complex networks. Nature 410, 268-276 (2001).

9. Milo, R. et al. Network motifs: Simple building blocks of complex networks. Science 298, 824-827 (2002).

10. Albert, R. \& Barabási, A. L. Statistical mechanics of complex networks. Rev. Mod. Phys. 74, 47-97 (2002).

11. Watts, D. J. A simple model of global cascades on random networks. Proc. Natl Acad. Sci. USA 99, 5766-5771 (2002).

12. Newman, M. E. J. The structure and function of complex networks. SIAM Rev. $45,167-256$ (2003)

13. Dorogovtsev, S. N. \& Mendes, J. F. F. Evolution of Networks: From Biological Nets to the Internet and WWW (Physics) (Oxford Univ. Press, 2003).

14. Bonanno, G., Caldarelli, G., Lillo, F. \& Mantegna, R. N. Topology of correlation-based minimal spanning trees in real and model markets. Phys. Rev. E 68, 046130 (2003).

15. Barrat, A., Barthelemy, M., Pastor-Satorras, R. \& Vespignani, A. The architecture of complex weighted networks. Proc. Natl Acad. Sci. USA 101, 3747-3752 (2004).

16. Newman, M. E. J. \& Girvan, M. Finding and evaluating community structure in networks. Phys. Rev. E 69, 026113 (2004).

17. Satorras, R. P. \& Vespignani, A. Evolution and Structure of the Internet: A Statistical Physics Approach (Cambridge Univ. Press, 2004).

18. Gallos, L. K., Cohen, R. \& Argyrakis, P. et al. Stability and topology of scale-free networks under attack and defense strategies. Phys. Rev. Lett. 94, 188701 (2005)

19. Song, C., Havlin, S. \& Makse, H. A. Self-similarity of complex networks. Nature 433, 392-395 (2005)

20. Boccaletti, S., Latora, V., Moreno, Y., Chavez, M. \& Hwang, D. U. Complex networks: Structure and dynamics. Phys. Rep. 424, 175-308 (2006).

21. Newman, M. E. J., Barabási, A-L. \& Watts, D. J. The Structure and Dynamics of Networks (Princeton Univ. Press, 2006).

22. Caldarelli, G. \& Vespignani, A. Large Scale Structure and Dynamics of Complex Webs (World Scientific, 2007).

23. Barrát, A., Barthélemy, M. \& Vespignani, A. Dynamical Processes on Complex Networks (Cambridge Univ. Press, 2008).

24. Cohen, R. \& Havlin, S. Complex Networks: Structure, Robustness and Function (Cambridge Univ. Press, 2010).

25. Kitsak, M. et al. Identification of influential spreaders in complex networks. Nature Phys. 6, 888-893 (2010).

26. Newman, M. E. J. Networks: An Introduction (Oxford Univ. Press, 2010).

27. Cohen, R. \& Havlin, S. Complex Networks: Structure, Robustness, and Function (Cambridge Univ. Press, 2010).

28. West, B. J. \& Grigolini, P. Complex Webs: Anticipating the Improbable (Cambridge Univ. Press, 2011).
29. Barthélemy, M. Spatial networks. Phys. Rep. 499, 1-101 (2011).

30. Li, D., Kosmidis, K., Bunde, A. \& Havlin, S. Dimension of spatially embedded networks. Nature Phys. 7, 481-484 (2011).

31. Snijders, T. A. B., Pattison, P. E., Robins, G. L. \& Handcock, M. S. New specifications for exponential random graph models. Sociol. Methodol. 36, 99-153 (2006).

32. Borgatti, S. P. Identifying sets of key players in a network. Comput. Math. Org. Theor. 12, 21-34 (2006).

33. Onnela, J-P. et al. Structure and tie strengths in mobile communication networks. Proc. Natl Acad. Sci. USA 104, 7332-7336 (2007).

34. Faust, K. \& Zvezki, M. Comparing social networks: Size, density and local structure. Linear Algebr. Appl. 3, 185-216 (2006).

35. Handcock, M. S., Raftery, A. E. \& Tantrum, J. M. Model-based clustering for social networks. J. R. Stat. Soc. A 170, 301-354 (2007).

36. Jackson, M. O. \& Rogers, B. W. Meeting strangers and friends of friends: How random are social networks? Am. Econom. Rev. 97, 890-915 (2007).

37. Kleinberg, J. The convergence of social and technological networks. Commun. ACM 51, 66-72 (2008).

38. Liben-Nowell, D. \& Kleinberg, J. Tracing information flow on a global scale using internet chain-letter data. Proc. Natl Acad. Sci. USA 105, 4633-4638 (2008).

39. Borgatti, S. P., Mehra, A., Brass, D. \& Labianca, G. Network analysis in the social sciences. Science 323, 892-895 (2009).

40. Joost, R. Inoperability input-output modeling of disruptions to interdependent economic systems. Syst. Eng. 9, 20-34 (2006).

41. Jackson, M. O. Social and Economic Networks (Economics, Physics, Sociology) (Princeton Univ. Press, 2008).

42. Zimmerman, R. Decision-making and the vulnerability of interdependent critical infrastructure. 2004 IEEE Int. Conf. Syst. Man Cybern. 5, 4059-4063 (2005).

43. Mendonca, D. \& Wallace, W. A. Impacts of the 2001 World Trade Center attack on New York City critical infrastructures. J. Infrast. Syst. 12, 260-270 (2006)

44. Robert, B., Morabito, L. \& Christie, R. D. The operational tools for managing physical interdependencies among critical infrastructures. Int. J. Crit. Infrastruct. 4, 353-367 (2008).

45. Reed, D. A., Kapur, K. C. \& Christie, R. D. Methodology for assessing the resilience of networked infrastructure. IEEE Syst. J. 3, 174-180 (2009).

46. Bagheri, E. \& Ghorbani, A. A. UML-CI: A reference model for profiling critical infrastructure systems. Inform. Syst. Front. 12, 115-139 (2009).

47. Mansson, D., Thottappillil, R., Backstrom, M. \& Ludvika, H. V. V. Methodology for classifying facilities with respect to intentional EMI. IEEE Trans. Electromagn. Compat. 95, 46-52 (2009).

48. Johansson, J. \& Hassel, H. An approach for modelling interdependent infrastructures in the context of vulnerability analysis. Reliab. Eng. Syst. Saf. $\mathbf{9 5}$ 1335-1344 (2010).

49. Alon, U. Biological networks: The tinkerer as an engineer. Science 301, 1866-1867 (2003)

50. Khanin, R. \& Wit, E. How scale-free are biological networks. J. Comput. Biol. $13,810-818$ (2006)

51. Colizza, V., Barrat, A., Barthelemy, M. \& Vespignani, A. Prediction and predictability of global epidemics: The role of the airline transportation network. Proc. Natl Acad. Sci. USA 103, 2015-2020 (2006).

52. Bunde, A. \& Havlin, S. Fractals and Disordered Systems (Springer, 1996).

53. Schneider, C. M., Araújo, N. A. M., Moreira, A. A., Havlin, S. \& Herrmann, H. J. Mitigation of malicious attacks on networks. Proc. Natl Acad. Sci. USA 108, 3838-3841 (2011).

54. Cohen, R., Havlin, S. \& Ben-Avraham, D. Efficient immunization strategies for computer networks and populations. Phys. Rev. Lett. 91, 247901 (2003).

55. Chen, Y., Paul, G., Havlin, S., Liljeros, F. \& Stanley, H. E. Finding a better immunization strategy. Phys. Rev. Lett. 101, 058701 (2008).

56. Braunstein, L. A., Buldyrev, S. V., Cohen, Havlin, S. \& Stanley, H. E. Optimal paths in disordered complex networks. Phys. Rev. Lett. 91, 168701 (2003).

57. Pastor-Satorras, R. \& Vespignani, A. Epidemic spreading in scale-free network Phys. Rev. Lett. 86, 3200-3203 (2001).

58. Balcan, D. et al. Multiscale mobility networks and the large scale spreading of infectious diseases. Proc. Natl Acad. Sci. USA 106, 21484-21489 (2009).

59. Palla, G., Derenyi, I., Farkas, I. \& Vicsek, T. Uncovering the overlapping community structure of complex networks in nature and society. Nature $\mathbf{4 3 5}$, 814-818 (2005)

60. Kossinets, G. \& Watts, D. Empirical analysis of an evolving social network. Science 311, 88-90 (2006).

61. Newman, M. E. J. The structure of scientific collaboration networks. Proc. Natl Acad. Sci. USA 98, 404-409 (2001).

62. Girvan, M. \& Newman, M. E. J. Community structure in social and biological networks. Proc. Natl Acad. Sci. USA 99, 7821-7826 (2002).

63. Moreira, A. A., Andrade, J. S. Jr, Herrmann, H. J. \& Indekeu, J. O. How to make a fragile network robust and vice versa. Phys. Rev. Lett. 102, 019701 (2009). 
64. Lopez, E., Buldyrev, S. V., Havlin, S. \& Stanley, H. E. Anomalous transport in scale-free networks. Phys. Rev. Lett. 94, 248701 (2005).

65. Boguñá, M. \& Krioukov, D. Navigating ultrasmall worlds in ultrashort time. Phys. Rev. Lett. 102, 058701 (2009).

66. Leicht, E. A. \& D'Souza, R. M. Percolation on interacting networks. Preprint at http://arxiv.org/abs/0907.0894. (2009).

67. Rosato, V. Modeling interdependent infrastructures using interacting dynamical models. Int. J. Crit. Infrastruct. 4, 63-79 (2008).

68. US-Canada Power System Outage Task Force Final Report on the August 14th 2003 Blackout in the United States and Canada: Causes and Recommendations (The Task Force, 2004).

69. Peerenboom, J., Fischer, R. \& Whitfield, R. in Proc. CRIS/DRM/IIIT/NSF Workshop Mitigating the Vulnerability of Critical Infrastructures to Catastrophic Failures (2001)

70. Rinaldi, S., Peerenboom, J. \& Kelly, T. Identifying, understanding, and analyzing critical infrastructure interdepedencies. IEEE Control. Syst. Magn. 21, 11-25 (2001).

71. Yağan, O., Qian, D., Zhang, J. \& Cochran, D. Optimal allocation of interconnecting links in cyber-physical systems: Interdependence, cascading failures and robustness. http://www.ece.umd.edu/ oyagan/Journals/ Interdependent_Journal.pdf (2011).

72. Vespignani, A. The fragility of interdependency. Nature 464, 984-985 (2010).

73. Buldyrev, S. V., Parshani, R., Paul, G., Stanley, H. E. \& Havlin, S. Catastrophic cascade of failures in interdependent networks. Nature 464, 1025-1028 (2010).

74. Newman, M. E. J., Strogatz, S. H. \& Watts, D. J. Random graphs with arbitrary degree distributions and their applications. Phys. Rev. E 64, 026118 (2001).

75. Shao, J., Buldyrev, S. V., Braunstein, L. A., Havlin, S. \& Stanley, H. E. Structure of shells in complex networks. Phys. Rev. E 80, 036105 (2009).

76. Parshani, R., Buldyrev, S. V. \& Havlin, S. Interdependent networks: Reducing the coupling strength leads to a change from a first to second order percolation transition. Phys. Rev. Lett. 105, 048701 (2010).

77. Huang, X., Gao, J., Buldyrev, S. V., Havlin, S. \& Stanley, H. E. Robustness of interdependent networks under targeted attack. Phys. Rev. E (R) 83, 065101 (2011).

78. Shao, J., Buldyrev, S. V., Havlin, S. \& Stanley, H. E. Cascade of failures in coupled network systems with multiple support-dependence relations. Phys. Rev. E 83, 036116 (2011).

79. Parshani, R., Rozenblat, C., Ietri, D., Ducruet, C. \& Havlin, S. Inter-similarity between coupled networks. Europhys. Lett. 92, 68002-68006 (2010).

80. Gu, C. et al. Onset of cooperation between layered networks. Phys. Rev. E 84, 026101 (2011)

81. Cho, W., Coh, K. \& Kim, I. Correlated couplings and robustness of coupled networks. Preprint at http://arxiv.org/abs/1010.4971. (2010).

82. Buldyrev, S. V., Shere, N. W. \& Cwilich, G. A. Interdependent networks with identical degrees of mutually dependent nodes. Phys. Rev. E 83, 016112 (2011).

83. Hu, Y., Ksherim, B., Cohen, R. \& Havlin, S. Percolation in interdependent and interconnected networks: Abrupt change from second to first order transition. Phys. Rev. E (in the press). Preprint at http://arxiv.org/abs/1106.4128 (2011).
84. Sachtjen, M. L., Carreras, B. A. \& Lynch, V. E. Disturbances in a power transmission system. Phys. Rev. E 61, 4877-4882 (2000).

85. Motter, A. E. \& Lai, Y. C. Cascade-based attacks on complex networks. Phys. Rev. E 66, 065102 (2002).

86. Moreno, Y., Pastor, S. R., Vázquez, A. \& Vespignani, A. Critical load and congestion instabilities in scale-free networks. Europhys. Lett. 62, 292-298 (2003).

87. Motter, A. E. Cascade control and defense in complex networks. Phys. Rev. Lett. 93, 098701 (2004).

88. Parshani, R., Buldyrev, S. V. \& Havlin, S. Critical effect of dependency groups on the function of networks. Proc. Natl Acad. Sci. USA 108, 1007-1010 (2011).

89. Bashan, A., Parshani, R. \& Havlin, S. Percolation in networks composed of connectivity and dependency links. Phys. Rev. E 83, 051127 (2011).

90. Bashan, A. \& Havlin, S. The combined effect of connectivity and dependency links on percolation of networks. J. Stat. Phys. 145, 686-695 (2011).

91. Molloy, M. \& Reed, B. The size of the giant component of a random graph with a given degree sequence. Combin. Probab. Comput. 7, 295-305 (1998).

92. Erdős, P. \& Rényi, A. On random graphs I. Publ. Math. 6, 290-297 (1959).

93. Erdős, P. \& Rényi, A. On the evolution of random graphs. Inst. Hung. Acad. Sci. 5, 17-61 (1960).

94. Bollobás, B. Random Graphs (Academic, 1985)

95. Schneider, C. M., Araújo, N. A. M., Havlin, S. \& Herrmann, H. J. Towards designing robust coupled networks. Preprint at http://arxiv.org/abs/ 1106.3234 (2011).

96. Gao, J., Buldyrev, S. V., Havlin, S. \& Stanley, H. E. Robustness of a network of networks. Phys. Rev. Lett. 107, 195701 (2011).

97. Gao, J, Buldyrev, S. V., Havlin, S. \& Stanley, H. E. Robustness of a tree-like network of interdependent networks. Preprint at http://arxiv.org/abs/1108.5515 (2011).

98. Suchecki, K. \& Holyst, J. A. Ising model on two connected Barabasi-Albert networks. Phys. Rev. E 74, 011122 (2006).

99. Donges, J. F., Schultz, H. C. H., Marwan, N., Zou, Y. \& Kurths, J. Investigating the topology of interacting networks. Eur. Phys. J. B (2011, in the press).

\section{Acknowledgements}

We thank R. Parshani for helpful discussions. We thank the DTRA (Defense Threat Reduction Agency) and the Office of Naval Research for support. J.G. also thanks the Shanghai Key Basic Research Project (grant no 09JC1408000) and the National Natural Science Foundation of China (grant no 61004088) for support. S.V.B. acknowledges the partial support of this research through the B. W. Gamson Computational Science Center at Yeshiva College. S.H. thanks the European EPIWORK project, Deutsche Forschungsgemeinschaft (DFG) and the Israel Science Foundation for financial support.

\section{Additional information}

The authors declare no competing financial interests. Reprints and permissions information is available online at http://www.nature.com/reprints. Correspondence and requests for materials should be addressed to H.E.S. 